\title{
Optimization of brain PET imaging for a multicentre trial: the French CATI experience
}

\author{
Marie-Odile Habert ${ }^{1,2,35^{*}}$, Sullivan Marie ${ }^{1,2}$, Hugo Bertin ${ }^{1,2}$, Moana Reynal ${ }^{1,2}$, Jean-Baptiste Martini ${ }^{1,2}$, \\ Mamadou Diallo ${ }^{1,2}$, Aurélie Kas ${ }^{1,2,3}$, the CATI Nuclear Medicine network and Régine Trébossen ${ }^{4}$
}

\footnotetext{
* Correspondence: habert@upmc.fr 'Sorbonne Universités, UPMC Univ Paris 06, CNRS, INSERM, Laboratoire d'Imagerie Biomédicale, F-75013 Paris, France

${ }^{2}$ Centre pour l'Acquisition et le Traitement des Images (www.cati-neuroimaging.com), Paris, France

Full list of author information is available at the end of the article
}

\begin{abstract}
Background: CATI is a French initiative launched in 2010 to handle the neuroimaging of a large cohort of subjects recruited for an Alzheimer's research program called MEMENTO. This paper presents our test protocol and results obtained for the 22 PET centres (overall 13 different scanners) involved in the MEMENTO cohort. We determined acquisition parameters using phantom experiments prior to patient studies, with the aim of optimizing PET quantitative values to the highest possible per site, while reducing, if possible, variability across centres.
\end{abstract}

Methods: Jaszczak's and 3D-Hoffman's phantom measurements were used to assess image spatial resolution (ISR), recovery coefficients $(R C)$ in hot and cold spheres, and signal-to-noise ratio (SNR). For each centre, the optimal reconstruction parameters were chosen as those maximizing ISR and RC without a noticeable decrease in SNR. Point-spread-function (PSF) modelling reconstructions were discarded. The three figures of merit extracted from the images reconstructed with optimized parameters and routine schemes were compared, as were volumes of interest ratios extracted from Hoffman acquisitions. The net effect of the 3D-OSEM reconstruction parameter optimization was investigated on a subset of 18 scanners without PSF modelling reconstruction.

Results: Compared to the routine parameters of the 22 PET centres, average $R C$ in the two smallest hot and cold spheres and average ISR remained stable or were improved with the optimized reconstruction, at the expense of slight SNR degradation, while the dispersion of values was reduced.

For the subset of scanners without PSF modelling, the mean RC of the smallest hot sphere obtained with the optimized reconstruction was significantly higher than with routine reconstruction. The putamen and caudate-to-white matter ratios measured on 3D-Hoffman acquisitions of all centres were also significantly improved by the optimization, while the variance was reduced.

Conclusions: This study provides guidelines for optimizing quantitative results for multicentric PET neuroimaging trials.

Keywords: Neurology, PET/CT, Quality assurance, Standardization, Multicentre trials

\section{Springer}

(c) 2016 Habert et al. Open Access This article is distributed under the terms of the Creative Commons Attribution 4.0 International License (http://creativecommons.org/licenses/by/4.0/), which permits unrestricted use, distribution, and reproduction in any medium, provided you give appropriate credit to the original author(s) and the source, provide a link to the Creative Commons license, and indicate if changes were made. 


\section{Background}

CATI is a French platform funded in 2010 with the aim of supporting multicentre clinical trials involving neuroimaging (cati-neuroimaging.com). The main project devoted to CATI was to handle the multimodality imaging aspect of a large cohort of subjects included in a research program on Alzheimer's disease (AD). This program, called MEMENTO, is a longitudinal study aiming at better understanding the natural history of Alzheimer's disease. It has enrolled 2300 subjects with either light cognitive deficits or cognitive complaints that will be followed up for at least 5 years (http://clinicaltrials.gov/show/NCT01926249).

The imaging aspect of the MEMENTO study includes the acquisition of PET cerebral glucose metabolism and amyloid distribution, with MRI studies on anatomy, diffusion, and resting state. In this paper, we focus on the optimization of PET quantitative values to the highest possible per site, while reducing, if possible, the variability across centres for the MEMENTO cohort. Phantom data were acquired during a technical visit to the PET centres, prior to patients' participation in the trial. The same phantom data were also acquired during follow-up visits after 18 months. The present study only addresses the data acquired during the site technical set-up.

Several large cohort studies on AD have been launched since the early 2000s. Among them, ADNI-1 was a forward-thinking, imaging-based multicentre clinical trial that involved 50 centres in North America, with the aim of identifying biomarkers of AD. For the PET imaging carried out in this study, the ADNI PET core determined reconstruction parameters for each scanner model and 3D-Hoffman phantoms were acquired with a standard protocol. However, discrepancies between the image characteristics from different PET centres remained high and were accounted for by degrading the spatial resolution to the lowest value among centres [1].

Other multicentre studies specifically focusing on AD have been conducted [2, 3]. In contrast to the ADNI trial, no phantom acquisition was performed prior to the study, and no post-filtering was applied to the data. Differences in spatially normalized FDGPET scans obtained with scanners of different resolutions were minimized by the following measures: (i) restricting the analysis to voxels with intensity $80 \%$ greater than the whole-brain mean, and (ii) excluding voxels from the uppermost 10 slices (i.e., from the top $22.5 \mathrm{~mm}$ of the brain) and from the lowermost 5 slices, where significant interscanner effects due to different fields of view have been reported [3].

The most advanced domain for PET image acquisition harmonization across multiple centres is oncological imaging. Guidelines for PET centres involved in clinical trials have been written by a group of experts, under the umbrella of the European society of Nuclear Medicine [4,5], or the American College of Radiology Imaging Network [6]. These guidelines are based on quality checks with phantoms reproducing the imaging conditions encountered in the abdomen and the thorax in glucose metabolism studies. In addition to these tomography quality checks, to ensure the highest reproducibility of the measures extracted from the image for patient follow-up, disease evolution, and recovery after treatments, these groups have formulated recommendations for data analysis. Overall, these recommendations enable the cross-comparison of measurements from different centres. According to our knowledge, the latest guidelines for FDG-PET brain imaging were published in 2009 [7] and do not meet our objectives. 
This paper presents our test protocol and the results obtained for the 22 PET centres involved in the MEMENTO cohort. French PET centres were equipped with systems set up between 2003 and 2012. No brain-dedicated high-resolution system or 2D tomography systems were included. Therefore, we chose to optimize acquisition and reconstruction parameters using phantom experiments prior to patient studies, with the aim of optimizing PET quantitative values to the highest possible per site, while reducing, if possible, the variability across centres. We also checked the impact of this harmonization on signal-to-noise ratios, as well as the exclusion of reconstruction with point-spread-function (PSF) modelling.

\section{Methods}

\section{Phantom studies}

Two phantom studies were acquired for the qualification process of the 22 centres, the Jaszczak phantom and the Hoffman 3D brain phantom. Standardized uptake value (SUV) measurements were also checked to assess the cross-calibration between the PET scanner and the dose calibration system.

The Deluxe Jaszczak phantom (model ECT/DLX/P) is a Plexiglas cylinder partially composed of cold rods (diameters 4.8 to $12.7 \mathrm{~mm}$ ) and partially of six inserted hollow spheres. The main cylinder of $6060 \mathrm{~mL}$ was filled with a $5 \mathrm{kBq} \mathrm{mL}{ }^{-1}$ FDG solution. Four spheres (internal diameters 7.86, 12.43, 15.43, and $24.82 \mathrm{~mm}$ ) were filled with a $15 \mathrm{kBq} \mathrm{mL}^{-1}$ FDG solution. The remaining two spheres (internal diameters 9.89 and $31.27 \mathrm{~mm}$ ) were filled with cold water.

The Hoffman 3D brain phantom (model BR/3D/P) was filled with a 37 -to- $55 \mathrm{kBq} \mathrm{mL}$ FDG solution, taking great care to avoid the presence of bubbles.

A dynamic acquisition of $3 \times 5$ min was performed for each phantom. CT acquisition parameters were set such that the effective dose would be low $(\leq 0.3 \mathrm{mSv})$, in accordance with the recommendations from the European Association of Nuclear Medicine [7].

The Hoffman phantom was positioned on a head holder when available, with the Deluxe Jaszczak phantom (20-30 kBq mL ${ }^{-1}$ FDG solution) below it for the simulation of a diffusion medium, as in subjects. Different reconstruction parameters were tested with varying reconstruction algorithms, number of iterations and subsets, reconstruction diameters, matrix sizes, or filters, always including those routinely used in brain studies. For the first 5 pilot centres, up to 10 sets of reconstructions were tested. Attenuation (derived from a CT scan) and scatter were corrected using standard software supplied by the scanner manufacturers.

Acquisition from a uniform cylinder filled with $5 \mathrm{kBq} \mathrm{mL}{ }^{-1}$ FDG solution was also performed to verify cross-calibration.

The same team of two technologists certified for radiation protection prepared all the phantoms. The CATI PET project manager supervised the whole procedure and the setting of reconstruction parameters.

\section{Analysis of the Deluxe Jaszczak phantom studies Jaszczak phantom studies}

The different image reconstruction schemes were compared based on recovery coefficients $(\mathrm{RC})$ computed for each sphere and spatial resolution estimated from the cold 
rods. $\mathrm{RC}$ were calculated from measurements in volumes of interest (VOIs) defined on each sphere and on the background. In-house software was developed for this purpose. It includes the following steps (all displayed for quality check):

1) For all sets of reconstructed PET volumes, the computation of the spheres' centre of gravity, based on an automatic segmentation of the spheres.

2) The computation of the spheres' centre of gravity based on the automatic segmentation of the spheres on CT scan volume, using the Hough algorithm [8], optimized with the incorporation of the spheres' specifications.

3) VOIs were defined using both the CT spheres' centre of gravity (determined in step 2) and the known sphere diameters, corrected if necessary for a mismatch between PET and CT spheres, then eroded to exclude the sphere wall. The background activity concentration was measured in VOIs obtained after the rotation of the VOIs drawn on the spheres.

4) The measurement of the mean activity in each sphere and background (BG), and calculation of $\mathrm{RC}\left(\mathrm{RC}_{\mathrm{H}}\right.$ and $\mathrm{RC}_{\mathrm{C}}$, respectively, for hot and cold spheres):

$$
\mathrm{RC}_{\mathrm{H}}=\frac{\left(M_{\mathrm{S}}-M_{\mathrm{BG}}\right) /\left(M_{\mathrm{S}}+M_{\mathrm{BG}}\right)}{\left(A_{\mathrm{S}}-A_{\mathrm{BG}}\right) /\left(A_{\mathrm{S}}+A_{\mathrm{BG}}\right)} \quad \mathrm{RC}_{\mathrm{C}}=\frac{\left(M_{\mathrm{BG}}-M_{\mathrm{S}}\right) /\left(M_{\mathrm{S}}+M_{\mathrm{BG}}\right)}{\left(A_{\mathrm{BG}}-A_{\mathrm{S}}\right) /\left(A_{\mathrm{S}}+A_{\mathrm{BG}}\right)},
$$

where $M_{\mathrm{S}}$ is the activity measured in the sphere, $M_{\mathrm{BG}}$ is the activity measured in the background, $A_{\mathrm{S}}$ is the actual activity injected in the sphere, and $A_{\mathrm{BG}}$ is the actual activity injected in the background.

5) Signal-to-noise ratio was calculated as follows:

$$
\mathrm{SNR}=\frac{M_{\mathrm{S}_{12.43}}-M_{\mathrm{BG}}}{\mathrm{SD}_{\mathrm{BG}}}
$$

where $M_{\mathrm{S} \_12.43}$ is the mean activity measured in the hot sphere of $12.43 \mathrm{~mm}, M_{\mathrm{BG}}$ is the mean activity measured in the background, and $\mathrm{SD}_{\mathrm{BG}}$ is the standard deviation in the background.

6) Display of the cold rod images and spheres for visual assessment of noise and spatial resolution, and of tables and graphics for all obtained $\mathrm{RC}$ according to the different reconstruction parameters (Fig. 1).

We also computed the image spatial resolution (ISR) using a method developed by Prieto et al. [9] to estimate the full width at half maximum (FWHM) of each PET scanner from the three largest hot spheres. The process can be summarized in three main steps:

1) Calculation of a ratio $R_{\text {mes }}$ as follows: 


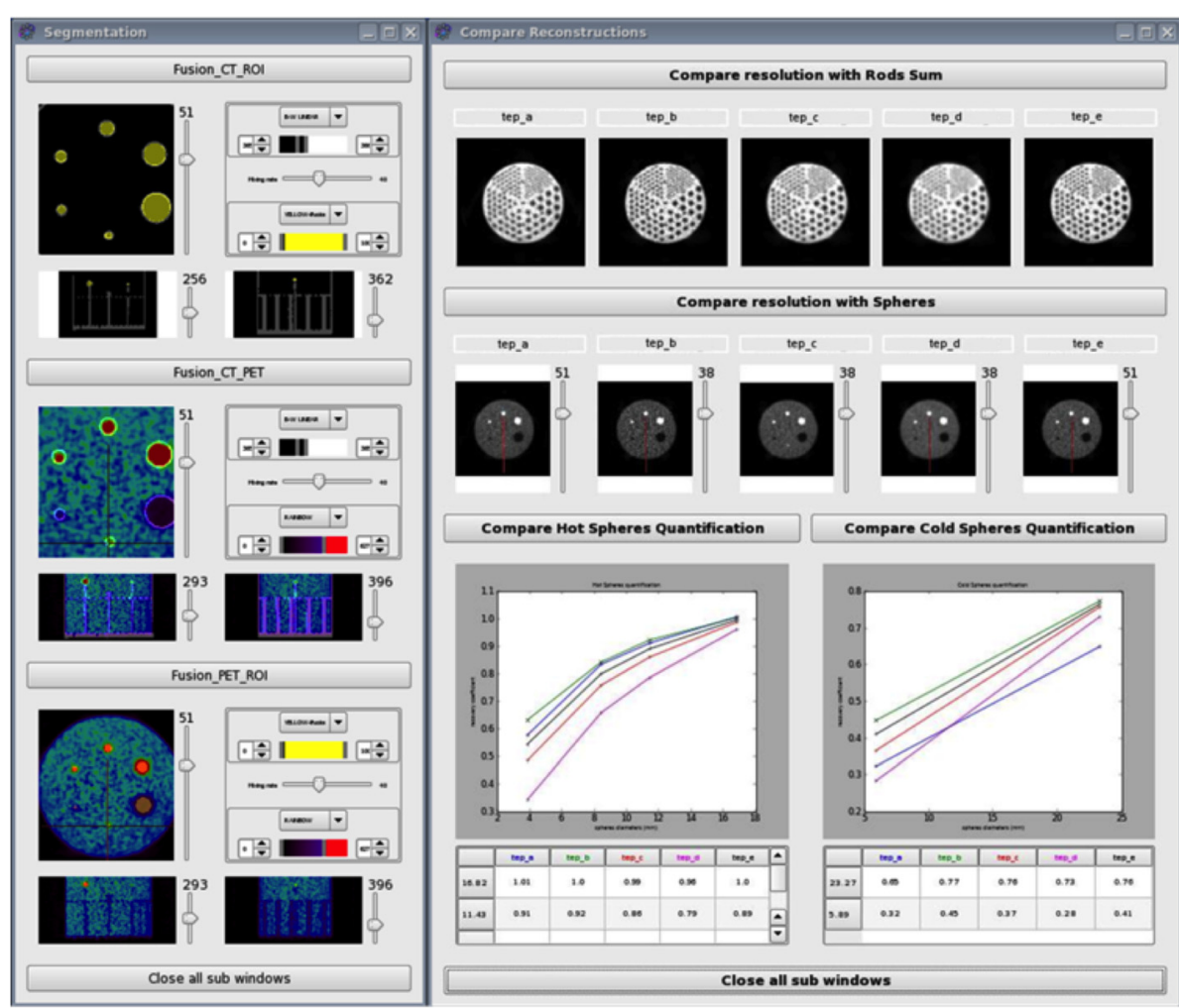

Fig. 1 In-house software interface showing the different steps allowing the building of volumes of interest in Jaszczak phantom spheres on the left, and the results from the different reconstruction parameters (named $a, b, c, d$, and e) tested on the right

$$
R_{\mathrm{mes}}=\frac{\operatorname{Max}_{\mathrm{S}}}{7 A_{\mathrm{S}}}
$$

where $\mathrm{Max}_{\mathrm{S}}$ is the maximum activity measured in the sphere and $A_{\mathrm{S}}$ is the actual activity injected in the hot spheres. Each $R_{\text {mes }}$ was normalized by the corresponding value of the largest sphere in order to limit the bias introduced by attenuation and scatter corrections.

2) Computation of a mask of the three spheres at the same position in the scanner field of view as during the acquisition; this mask was then convolved with a threedimensional isotropic Gaussian function with different FWHM values ranging from 4 to $10 \mathrm{~mm}$ and a step of $0.1 \mathrm{~mm}$. A theoretical ratio $R_{\text {the }}$ was then calculated for each of the three spheres as follows:

$$
R_{\text {the }}=\frac{\operatorname{Max}_{\mathrm{S}}}{A_{\mathrm{S}}}
$$

where $\mathrm{Max}_{\mathrm{S}}$ is the maximum value measured in the sphere after convolution and $A_{\mathrm{S}}$ is the actual value. 
3) The optimal ISR value was selected as the FWHM value that best fitted the experimental values, according to minimization of the normalized sum of squared differences between observed $R_{\text {mes }}$ and simulated $R_{\text {the }}$ over the three spheres.

\section{Analysis of the Hoffman 3D brain phantom studies}

All reconstructed volumes were co-registered to the highest quality CT scan of the phantom (acquired at centre no. 16) using SPM8. The grey matter ribbon was obtained by thresholding CT image intensity at $60 \%$ of the maximum value. The CT scan was also manually segmented in volumes of interest (Fig. 2) in order to compute right-to-left (R/L) and anterior-to-posterior $(\mathrm{L} / \mathrm{P})$ cortical ratios, as well as caudate-to-white matter (C/ WM), putamen-to-white matter (P/WM), and grey-to-white matter (GM/WM) ratios from PET images. The upper and lower slices were discarded for the analyses.

\section{Image reconstruction}

First, the three frames were summed. We then systematically compared phantom measurements obtained from images reconstructed with the algorithm selected for diagnostic purposes by each centre with the images reconstructed using the parameters optimized by CATI. Reconstruction algorithms incorporating the modelling of the spatial resolution of the tomographs were used for diagnostic purposes in four (4/22) centres. These algorithms were discarded by CATI to avoid possible additional centre

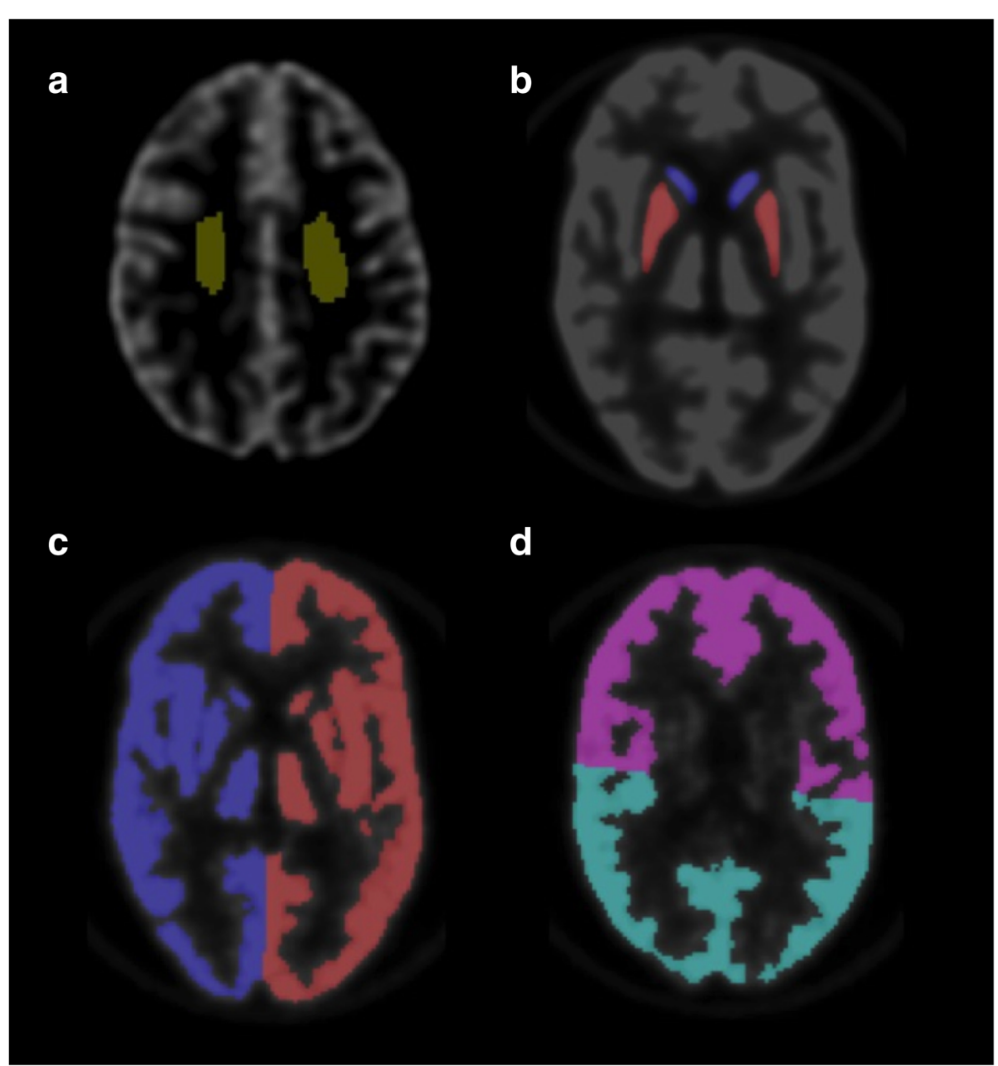

Fig. 2 Volumes of interest used for analysis of the Hoffman 3D brain phantom acquisitions. a White matter. b Caudate (blue) and putamen (red). c Right and left cortex. d Anterior and posterior cortex 
effects, and 3D-OSEM statistical image reconstruction algorithms, or FORE 2D-OSEM when the 3D-OSEM was not available, were selected.

The optimization was based on three figures of merit extracted from measurements on a Jaszczak phantom, the RC of spheres of various diameters, the signal-tobackground-noise ratio (SNR) and the ISR. The image reconstruction parameters were chosen at each site as a compromise between maximized RC values, ISR, and SNR. Volumes of interest ratios extracted from Hoffman acquisitions were also compared to confirm the choice of reconstruction parameters for each site. Our approach can be described as follows:

1) Reconstruction matrix was set to obtain pixel spacing inferior to $3 \mathrm{~mm}$

2) The number of total iterations multiplied by the number of subsets was chosen such as the product iterations $\times$ subsets was superior to 50 , and optimized with post-reconstruction smoothing

3) RC and ISF obtained with different combinations of reconstruction parameters were compared first: the parameters giving highest values with acceptable SNR were chosen.

4) Hoffmann images were then quantitatively and visually checked for the best compromise between spatial resolution and noise.

5) The optimized quantitative values for both phantoms obtained at each centre were finally compared to the routine values.

The optimized parameters chosen according to the model of scanner are presented in Table 1 .

\section{Statistical analyses}

The Wilcoxon signed rank test was used to compare routine and optimized values obtained from phantoms' studies. Variances were compared with a Pitman test [10]. A significance threshold of 0.05 was adopted for all statistical analyses. Statistical analyses were performed for all centres and also for a subset of 18 centres where PSF modelling reconstruction was not available in order to assess the impact of PSF modelling on the final optimization.

\section{Results}

All tomographs were 3D PET/CT, installed between 2003 and 2012, and consisted of 9 GE, 3 Philips, and 10 Siemens systems, with a total of 13 different models of scanners (Table 1).

In four centres (nos. 8, 13, 19, and 22), an error greater than $10 \%$ was found for cross-calibration measurements and was later corrected by the centre's physicist.

Four other scanners (nos. 10, 11, 14, and 20) presented a mismatch of more than $3 \mathrm{~mm}$ between CT and PET images, which required fixing by servicing the scanners.

\section{Jaszczak phantom results}

For all centres, the optimized $\mathrm{RC}$ of the two smallest hot spheres ranged from 0.17 to 0.52 (mean \pm SD $0.33 \pm 0.09$ ) and from 0.56 to 0.86 (mean \pm SD $0.71 \pm 0.08$ ), respectively 
Table 1 Optimized parameters chosen according to the model of the scanner

\begin{tabular}{|c|c|c|c|c|c|c|c|c|c|}
\hline GE models & Number & Installation year & Slice thickness (mm) & Recons. method & $\begin{array}{l}\text { Number of iterations } \\
\text { (iterations } \times \text { subsets) }\end{array}$ & $\begin{array}{l}\text { Post-reconstruction filter type } \\
\text { and FWHM if relevant (mm) }\end{array}$ & Matrix & $\begin{array}{l}\text { Pixel spacing } \\
(\mathrm{mm} \times \mathrm{mm})\end{array}$ & Others \\
\hline Discovery 690 & 3 & $2009 / 2010 / 2011$ & 3.27 & VPHD & $10 \times 36$ & Gaussian 3.5 & $256 \times 256$ & $1.17 \times 1.17$ & Z-filter: standard \\
\hline Discovery RX & 2 & 2009 & 3.27 & FORE + OSEM 2D & $7 \times 35$ & Gaussian 3.5 & $128 \times 128$ & $2.34 \times 2.34$ & $\begin{array}{l}\text { Loop filter: } 2.34 \mathrm{~mm} \\
\text { Z-filter: standard }\end{array}$ \\
\hline Discovery DST - E & 1 & 2004 & 3.27 & FORE + OSEM 2D & $5 \times 35$ & Gaussian 2.57 & $128 \times 128$ & $2.34 \times 2.34$ & $\begin{array}{l}\text { Loop filter: } 2.34 \mathrm{~mm} \\
\text { Z-filter: standard }\end{array}$ \\
\hline Discovery ST 4 & 1 & 2004 & 3.27 & FORE + OSEM 2D & $7 \times 35$ & Gaussian 3 & $256 \times 256$ & $1.17 \times 1.17$ & $\begin{array}{l}\text { Loop filter: } 2 \mathrm{~mm} \\
\text { Z-filter: standard }\end{array}$ \\
\hline Discovery ST & 2 & $2003 / 2004$ & 3.27 & FORE + OSEM 2D & $7 \times 32$ & Gaussian 2 or 2.57 & $128 \times 128$ & $2.34 \times 2.34$ & Loop filter: $2.34 \mathrm{~mm}$ \\
\hline Philips models & Number & Installation year & Slice thickness (mm) & Recons. method & $\begin{array}{l}\text { Number of iterations } \\
\text { (iterations } \times \text { subsets) }\end{array}$ & Smoothing (mm) & Matrix & $\begin{array}{l}\text { Pixel spacing } \\
(\mathrm{mm} \times \mathrm{mm})\end{array}$ & Others \\
\hline Gemini TF & 2 & $2008 / 2010$ & 2 & LOR - RAMLA & $10 \times 33$ & Smooth B & $128 \times 128$ & $2 \times 2$ & - \\
\hline Gemini GXL & 1 & 2006 & 2 & LOR - RAMLA & $10 \times N A$ & Smooth & $128 \times 128$ & $2 \times 2$ & - \\
\hline Siemens models & Number & Installation year & Slice thickness (mm) & Recons. method & $\begin{array}{l}\text { Number of iterations } \\
\text { (iterations } \times \text { subsets) }\end{array}$ & $\begin{array}{l}\text { Post-reconstruction } \\
\text { filter type and FWHM } \\
\text { if relevant }(\mathrm{mm})\end{array}$ & Matrix & $\begin{array}{l}\text { Pixel spacing } \\
(\mathrm{mm} \times \mathrm{mm})\end{array}$ & Others \\
\hline Biograph mCT 40/64 & 3 & $2009 / 2012$ & 2.027 & OSEM 3D & $12 \times 24$ & Gaussian 3 or 4 & $256 \times 256$ & $1.59 \times 1.59$ & - \\
\hline Biograph Hirez TruePoint & 1 & 2008 & 3 & OSEM 3D & $8 \times 21$ & Gaussian 4 & $336 \times 336$ & $1.02 \times 1.02$ & - \\
\hline Biograph 6 VB 20B True V & 1 & 2006 & 2 & FORE + OSEM 2D & $8 \times 21$ & Gaussian 4 & $256 \times 256$ & $1.34 \times 1.34$ & - \\
\hline Biograph 6 & 2 & $2004 / 2005$ & 2 & FORE + OSEM 2D & $8 \times 24$ & Gaussian 2 or 3 & $256 \times 256$ & $1.33 \times 1.33$ & - \\
\hline Biograph 16 VB40B & 1 & 2004 & 2 & FORE + OSEM 2D & $8 \times 24$ & Gaussian 3 & $336 \times 336$ & $1.02 \times 1.02$ & - \\
\hline Biograph LSO DUO & 2 & $2003 / 2004$ & 3.375 & FORE + OSEM 2D & $8 \times 16$ & Gaussian 2 & $256 \times 256$ & $1.33 \times 1.33$ & - \\
\hline
\end{tabular}

NA non applicable, OSEM Ordered Subset Expectation Maximization, VPHD VUE Point High Definition, LOR-RAMLA Line-of-Response Row-Action Maximum Likelihood Algorithm, FORE Fourier Rebinning 
(Fig. 3). Mean RCs were not significantly different for routine and optimized reconstructions, but the variance was significantly reduced ( $p=0.008$ and $p=0.002$, respectively). The optimized $\mathrm{RC}$ of the two cold spheres ranged from 0.22 to 0.52 (mean \pm SD $0.41 \pm 0.08$ ) for the smallest and from 0.60 to 0.84 for the largest (mean \pm SD $0.77 \pm$ $0.05)$. Mean RC significantly increased with optimized reconstructions $(p=0.003$ and $p=$ 0.004 , respectively) (Fig. 4). Variance was also significantly reduced for the largest sphere $(p=0.002)$. All RC values obtained with routine and optimized parameters are presented in Table 2, with the exception of four missing data from one centre, because of an operating error.

For the 18 centres where PSF modelling reconstruction was not available, the optimized RC of the two smallest hot spheres ranged from 0.17 to 0.52 (mean \pm SD $0.31 \pm$ 0.09 ) and from 0.56 to 0.86 (mean \pm SD $0.70 \pm 0.08$ ), respectively (Fig. 3). Mean RC of the smallest hot sphere was significantly higher for optimized reconstructions $p=0.04$ ), while the dispersion of values remained equivalent for both spheres. The optimized RC of the two cold spheres ranged from 0.22 to 0.52 (mean \pm SD $0.40 \pm 0.08$ ) for the smallest and from 0.60 to 0.84 for the largest (mean \pm SD $0.76 \pm 0.05$ ) (Fig. 4). Mean RC significantly increased with optimized reconstructions $(p=0.00002$ and $p=0.001$, respectively), but no significant difference was found for variances.

No significant difference could be detected $(p=0.61)$ between the values of the ratio "measured background/actual background" for routine reconstruction parameters $(0.97 \pm 0.13)$ and optimized reconstruction parameters $(0.99 \pm 0.08)$. In all centres, the SNR decreased between routine and optimized parameters, from $14.2 \pm 6.16$ to $9.74 \pm$

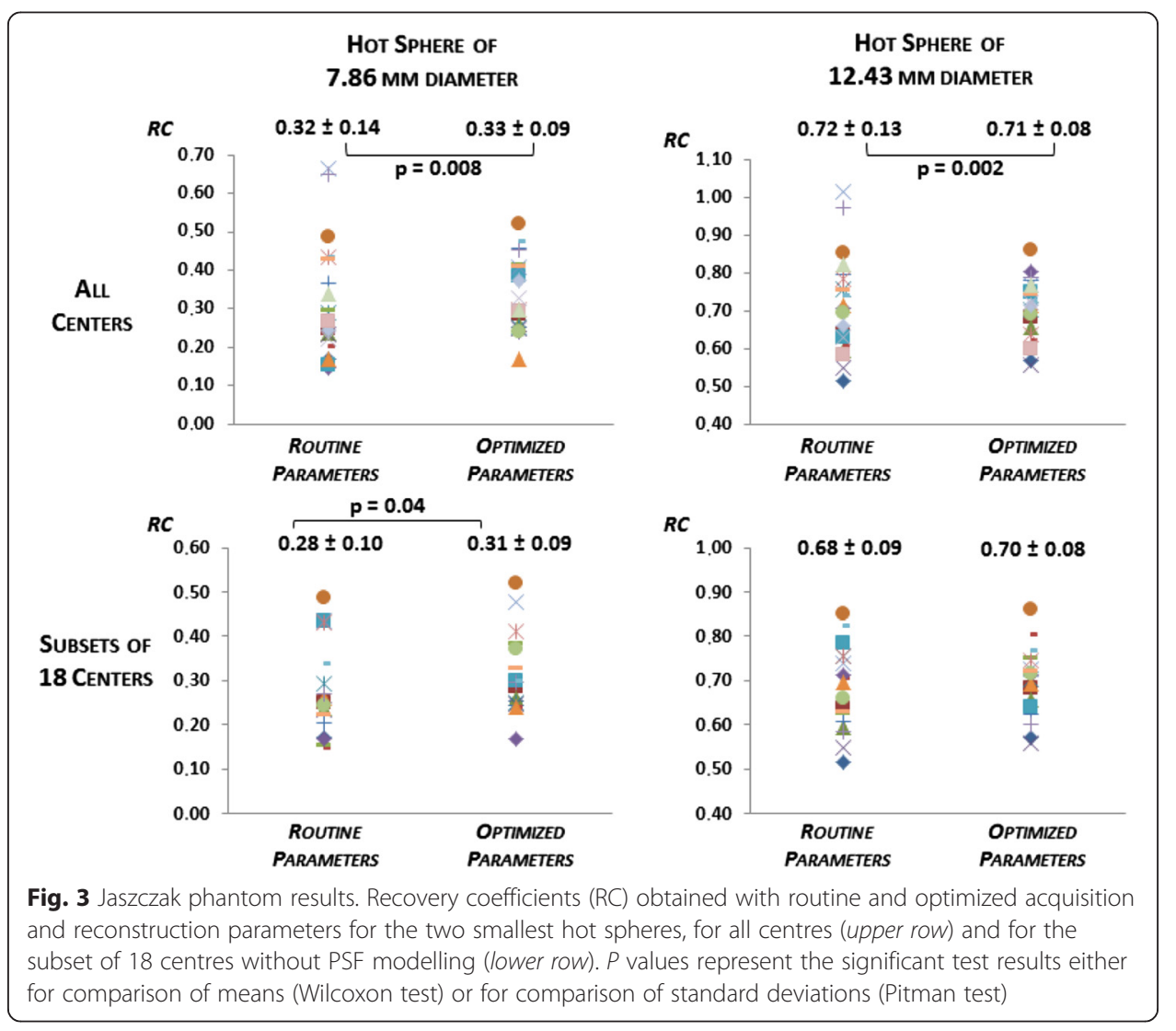




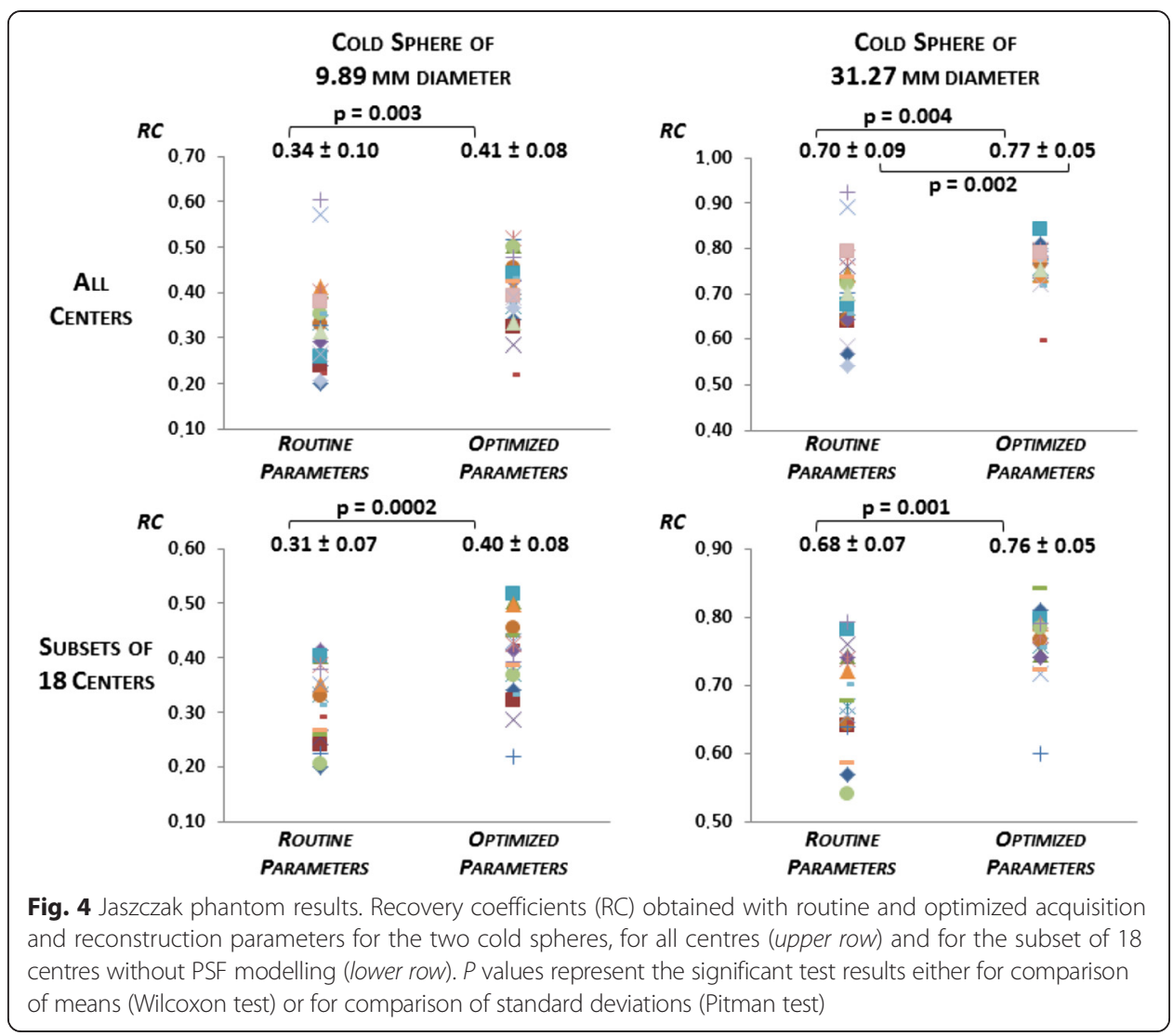

2.41 (mean $\pm \mathrm{SD}$ ). In the subset of 18 centres, it also decreased from $12.67 \pm 4.52$ to $9.70 \pm 2.5($ mean $\pm \mathrm{SD})$.

\section{Image spatial resolution measurements}

For all centres, the ISR ranged from 5.9 to $7.7 \mathrm{~mm}$ (mean \pm SD $6.76 \pm 0.6$ ) with optimized parameters and from 5.5 to $7.7 \mathrm{~mm}$ (mean \pm SD $6.98 \pm 0.6$ ) with routine parameters and did not differ significantly.

\section{D Hoffman phantom results}

For all centres, a significant increase of the mean C/WM $(p=0.005)$ and P/WM $(p=$ 0.04) ratios was observed between routine and optimized reconstructions, but not for the other ratios. The variance was significantly reduced for the GM/WM, C/WM, and $\mathrm{P} / \mathrm{WM}$ ratios ( $p=0.001 ; p=0.005 ; p=0.006$, respectively) (Fig. 5$)$. In the subset of 18 centres, a significant increase of both mean C/WM $(p=0.0002)$ and $\mathrm{P} / \mathrm{WM}$ ratios $(p=$ 0.006) was observed. Variances were not significantly different. The ratio values for both reconstructions and all centres are presented in Table 3.

\section{Discussion}

Multicentre clinical trials involving imaging require procedures for image acquisition and reconstruction to be optimized to account for inter-subject profile variability and to ensure the robustness of the analysis after pooling the data and for patient follow- 
Table 2 Recovery coefficient values for both reconstructions and all centres obtained from Jaszczak acquisitions

\begin{tabular}{|c|c|c|c|c|c|c|c|c|c|c|c|c|}
\hline \multirow[t]{2}{*}{$\begin{array}{l}\text { Centre } \\
\text { number }\end{array}$} & \multicolumn{2}{|c|}{$\begin{array}{l}7.86 \text { mm } \\
\text { Hot sphere }\end{array}$} & \multicolumn{2}{|c|}{$\begin{array}{l}12.43 \mathrm{~mm} \\
\text { Hot sphere }\end{array}$} & \multicolumn{2}{|c|}{$\begin{array}{l}14.43 \mathrm{~mm} \\
\text { Hot sphere }\end{array}$} & \multicolumn{2}{|c|}{$\begin{array}{l}24.82 \mathrm{~mm} \\
\text { Hot sphere }\end{array}$} & \multicolumn{2}{|c|}{$\begin{array}{c}9.89 \mathrm{~mm} \\
\text { Cold sphere }\end{array}$} & \multicolumn{2}{|c|}{$\begin{array}{l}24.82 \mathrm{~mm} \\
\text { Cold sphere }\end{array}$} \\
\hline & $R P$ & $O P$ & $R P$ & $O P$ & $R P$ & $O P$ & $R P$ & $\overline{O P}$ & $R P$ & $O P$ & $R P$ & $O P$ \\
\hline 1 & 0.17 & 0.25 & 0.52 & 0.57 & 0.56 & 0.59 & 0.74 & 0.74 & 0.20 & 0.34 & 0.57 & 0.81 \\
\hline 2 & 0.25 & 0.29 & 0.64 & 0.68 & 0.76 & 0.77 & 0.95 & 0.95 & 0.24 & 0.32 & 0.64 & 0.79 \\
\hline 3 & 0.24 & 0.26 & 0.59 & 0.66 & 0.70 & 0.75 & 0.91 & 0.92 & 0.40 & 0.50 & 0.74 & 0.74 \\
\hline 4 & 0.23 & 0.25 & 0.55 & 0.56 & 0.74 & 0.73 & 0.93 & 0.91 & 0.25 & 0.29 & 0.76 & 0.75 \\
\hline 5 & 0.29 & 0.25 & 0.76 & 0.70 & 0.78 & 0.74 & 0.94 & 0.92 & 0.33 & 0.37 & 0.67 & 0.76 \\
\hline 6 & 0.49 & 0.52 & 0.85 & 0.86 & 0.90 & 0.91 & 1.01 & 1.01 & 0.33 & 0.46 & 0.64 & 0.77 \\
\hline 7 & 0.37 & 0.46 & 0.79 & 0.78 & 0.89 & 0.82 & 1.04 & 0.97 & 0.33 & 0.52 & 0.70 & 0.78 \\
\hline 8 & 0.20 & 0.24 & 0.61 & 0.62 & 0.70 & 0.70 & 0.92 & 0.91 & 0.22 & 0.22 & 0.64 & 0.60 \\
\hline 9 & 0.30 & 0.41 & NA & NA & 0.87 & 0.82 & 1.06 & 0.99 & NA & NA & 0.73 & 0.80 \\
\hline 10 & 0.15 & 0.24 & 0.71 & 0.80 & 0.80 & 0.85 & 0.99 & 0.99 & 0.29 & 0.42 & 0.64 & 0.78 \\
\hline 11 & 0.15 & 0.38 & 0.63 & 0.75 & 0.68 & 0.78 & 0.92 & 0.95 & 0.26 & 0.44 & 0.68 & 0.84 \\
\hline 12 & 0.17 & 0.17 & 0.71 & 0.71 & 0.73 & 0.73 & 0.91 & 0.91 & 0.41 & 0.41 & 0.74 & 0.74 \\
\hline 13 & 0.67 & 0.41 & 1.02 & 0.77 & 0.97 & 0.79 & 1.03 & 0.94 & 0.57 & 0.41 & 0.89 & 0.78 \\
\hline 14 & 0.44 & 0.30 & 0.78 & 0.64 & 0.72 & 0.70 & 0.93 & 0.94 & 0.40 & 0.52 & 0.78 & 0.80 \\
\hline 15 & 0.26 & 0.24 & 0.70 & 0.69 & 0.75 & 0.73 & 0.93 & 0.92 & 0.35 & 0.50 & 0.72 & 0.79 \\
\hline 16 & 0.65 & 0.45 & 0.97 & 0.79 & 0.95 & 0.84 & 0.98 & 0.98 & 0.60 & 0.48 & 0.92 & 0.79 \\
\hline 17 & 0.43 & 0.48 & 0.74 & 0.73 & 0.78 & 0.77 & 0.95 & 0.94 & 0.35 & 0.43 & 0.66 & 0.72 \\
\hline 18 & 0.43 & 0.41 & 0.76 & 0.74 & 0.78 & 0.76 & 0.87 & 0.86 & 0.39 & 0.43 & 0.74 & 0.77 \\
\hline 19 & 0.25 & 0.37 & 0.66 & 0.72 & 0.68 & 0.73 & 0.92 & 0.93 & 0.21 & 0.37 & 0.54 & 0.78 \\
\hline 20 & 0.27 & 0.30 & 0.58 & 0.60 & 0.75 & 0.76 & 0.97 & 0.97 & 0.38 & 0.39 & 0.79 & 0.79 \\
\hline 21 & 0.34 & 0.30 & 0.82 & 0.77 & 0.84 & 0.80 & 1.00 & 0.99 & 0.31 & 0.33 & 0.70 & 0.76 \\
\hline 22 & 0.22 & 0.33 & 0.63 & 0.72 & 0.76 & 0.82 & 0.96 & 0.97 & 0.27 & 0.39 & 0.59 & 0.72 \\
\hline Mean & 0.32 & $0.33^{* *}$ & 0.72 & 0.71 & 0.78 & 0.77 & 0.95 & 0.94 & 0.34 & $0.41^{* * *}$ & 0.70 & $0.77^{* * *}$ \\
\hline S.D. & 0.14 & $0.09^{* * *}$ & 0.13 & $0.08^{* * *}$ & 0.09 & $0.06^{* * *}$ & 0.07 & 0.06 & 0.10 & 0.08 & 0.09 & $0.05^{* * *}$ \\
\hline
\end{tabular}

up. This phantom study proposed and validated a strategy for optimizing PET scanners for multicentre cerebral studies while reducing, but not minimizing, variability across centres. It involved 22 PET centres with 13 different PET/CT scanner models, most of which were of recent generation. We ensured the reproducibility of phantom measurements by sending the same technologists to the PET centres for scanner set-up.

We checked the cross-calibration between the tomograph and the dose calibration device and found a difference of more than $10 \%$ for four centres, which was immediately corrected. The alignment between the $\mathrm{CT}$ and PET scanners was also systematically checked and a correction was performed if necessary. Such a correction was needed in four additional centres.

\section{Choice of reconstruction parameters}

Harmonization across scanners and centres for multicentre cerebral imaging trials was one of the achievements of a previous study by the ADNI [11]. For that study, which 


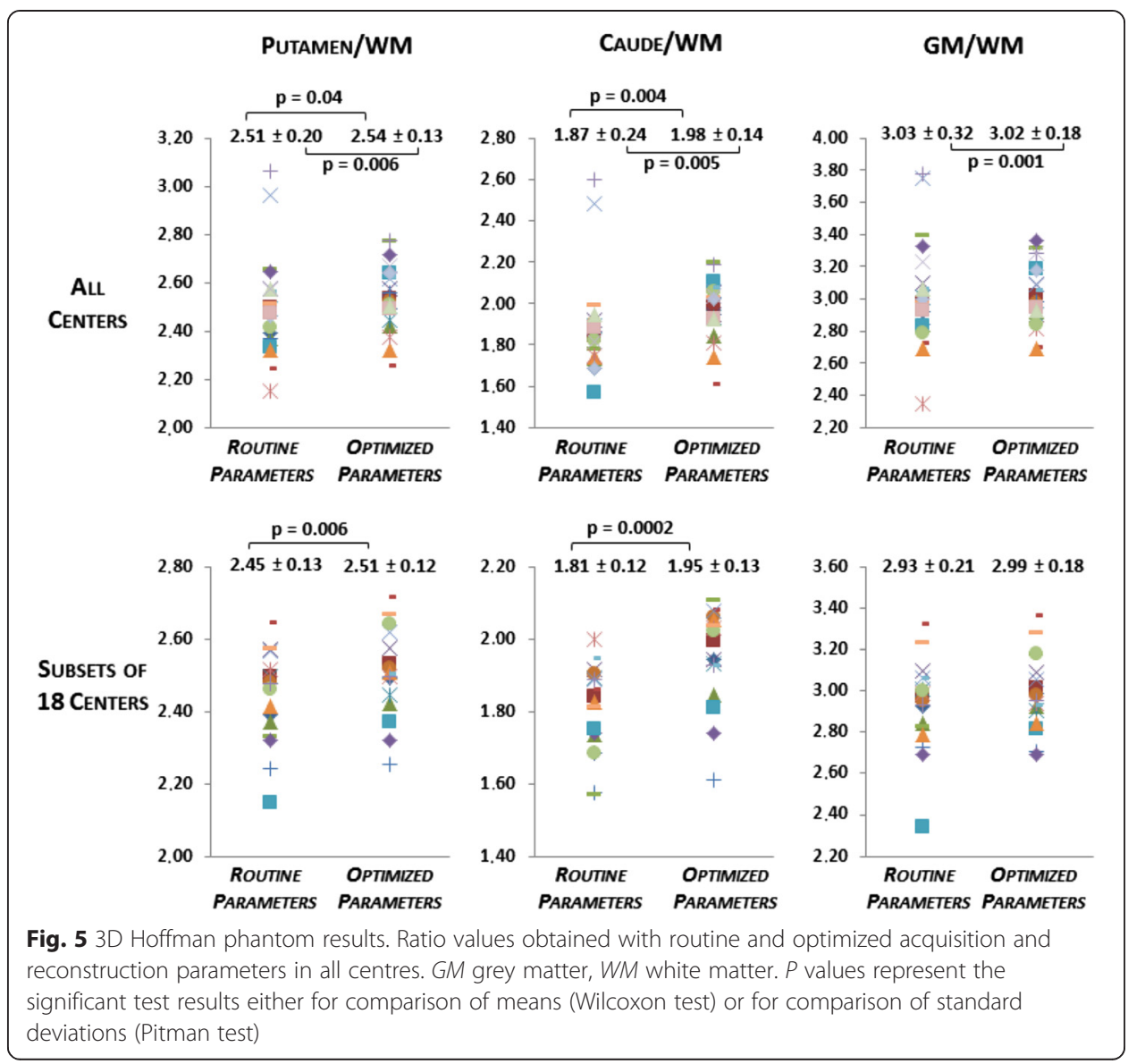

included 50 centres and 17 different PET scanners, the PET centres were asked to acquire two 3D-Hoffman studies with recommended parameters. The ADNI qualitycheck team then checked the phantom images. For the analysis of the pooled images, a post-reconstruction smoothing filter, determined from phantom measurements, was applied to the images. This filter aimed at homogenizing the spatial resolution of the images across centres, and its application translated to a degradation of the resolution to the lowest one encountered [1].

In the present study, we chose to optimize the reconstruction parameters (with a product iterations $\times$ subsets superior to 50 ) and the post-reconstruction filter so that the recovery coefficients in the small cold and hot spheres would reach an optimized mean value and present limited dispersion around this optimal value. To this end, we reconstructed the images using a conventional 3D algorithm with a description of the statistics of the recorded data only, although PSF modelling reconstructions were available on the scanners that were of the more recent generations.

As expected, the reconstructions with PSF modelling provided recovery coefficients closer to 1 in the two smallest hot and cold spheres than the reconstructions without resolution modelling. However, Gibbs artefacts [12] were detected on the images at the edges of spherical objects. We therefore discarded them to avoid these artefacts and also to limit the discrepancies in $\mathrm{RC}$ in images reconstructed with and without resolution modelling across centres. On the other hand, we allowed algorithms with timeof-flight (TOF) modelling in two centres. It is known that state-of-the-art TOF values 
Table 3 Ratio values for both reconstructions and all centres obtained from Hoffman-3D acquisitions

\begin{tabular}{|c|c|c|c|c|c|c|c|c|c|c|}
\hline \multirow{2}{*}{$\begin{array}{l}\text { Centre } \\
\text { number }\end{array}$} & \multicolumn{2}{|c|}{ Right/left ratio } & \multicolumn{2}{|c|}{ Anterior/posterior ratio } & \multicolumn{2}{|c|}{ Putamen/WM ratio } & \multicolumn{2}{|c|}{ Caudate/WM ratio } & \multicolumn{2}{|c|}{ GM/WM ratio } \\
\hline & $R P$ & $O P$ & $R P$ & $O P$ & $R P$ & $O P$ & $R P$ & $O P$ & $R P$ & $O P$ \\
\hline 1 & 1.01 & 1.00 & 1.00 & 1.01 & 2.39 & 2.49 & 1.68 & 1.95 & 2.92 & 2.98 \\
\hline 2 & 1.00 & 1.00 & 1.01 & 1.01 & 2.50 & 2.53 & 1.84 & 2.00 & 2.98 & 3.02 \\
\hline 3 & 1.01 & 1.01 & 1.00 & 1.00 & 2.37 & 2.42 & 1.73 & 1.85 & 2.84 & 2.92 \\
\hline 4 & 1.01 & 1.01 & 1.01 & 1.01 & 2.57 & 2.57 & 1.92 & 1.94 & 3.09 & 3.09 \\
\hline 5 & 0.99 & 0.99 & 1.02 & 1.02 & 2.50 & 2.45 & 1.89 & 1.93 & 3.01 & 2.91 \\
\hline 6 & 1.02 & 1.02 & 1.03 & 1.03 & 2.48 & 2.52 & 1.91 & 2.06 & 2.96 & 2.98 \\
\hline 7 & 1.00 & 1.00 & 1.05 & 1.01 & 2.37 & 2.56 & 1.71 & 2.03 & 2.85 & 2.98 \\
\hline 8 & 1.02 & 1.02 & 1.04 & 1.05 & 2.24 & 2.25 & 1.57 & 1.61 & 2.73 & 2.70 \\
\hline 9 & 1.01 & 1.01 & 1.01 & 1.01 & 2.66 & 2.77 & 1.78 & 2.20 & 3.40 & 3.32 \\
\hline 10 & 1.03 & 1.03 & 1.00 & 1.00 & 2.65 & 2.72 & 1.86 & 2.08 & 3.33 & 3.37 \\
\hline 11 & 1.03 & 1.03 & 0.98 & 0.97 & 2.33 & 2.64 & 1.57 & 2.11 & 2.83 & 3.18 \\
\hline 12 & 1.01 & 1.01 & 1.03 & 1.03 & 2.32 & 2.32 & 1.74 & 1.74 & 2.69 & 2.69 \\
\hline 13 & 1.00 & 1.00 & 1.05 & 1.04 & 2.96 & 2.62 & 2.48 & 2.05 & 3.75 & 3.09 \\
\hline 14 & 1.01 & 1.00 & 1.05 & 0.99 & 2.15 & 2.37 & 1.75 & 1.81 & 2.34 & 2.82 \\
\hline 15 & 1.00 & 1.00 & 1.01 & 1.01 & 2.42 & 2.51 & 1.83 & 2.05 & 2.79 & 2.84 \\
\hline 16 & 0.99 & 1.00 & 1.02 & 1.03 & 3.06 & 2.78 & 2.60 & 2.19 & 3.77 & 3.28 \\
\hline 17 & 1.00 & 1.00 & 1.01 & 1.02 & 2.57 & 2.62 & 1.89 & 2.08 & 3.06 & 3.06 \\
\hline 18 & 1.00 & 1.00 & 1.05 & 1.05 & 2.52 & 2.50 & 2.00 & 2.03 & 2.99 & 2.94 \\
\hline 19 & 0.99 & 0.99 & 1.01 & 1.02 & 2.46 & 2.64 & 1.68 & 2.02 & 3.00 & 3.18 \\
\hline 20 & 1.00 & 1.00 & 1.01 & 1.01 & 2.48 & 2.50 & 1.89 & 1.93 & 2.93 & 2.95 \\
\hline 21 & 1.00 & 1.00 & 1.02 & 1.02 & 2.58 & 2.51 & 1.95 & 1.93 & 3.06 & 2.93 \\
\hline 22 & 1.03 & 1.02 & 1.00 & 1.01 & 2.57 & 2.67 & 1.82 & 2.04 & 3.23 & 3.28 \\
\hline Mean & 1.01 & 1.01 & 1.02 & 1.02 & 2.51 & $2.54^{* * *}$ & 1.87 & $1.98^{* * *}$ & 3.03 & 3.02 \\
\hline S.D. & 0.01 & 0.01 & 0.02 & 0.02 & 0.20 & $0.13^{* * *}$ & 0.24 & $0.14^{* * *}$ & 0.32 & $0.18^{* * * *}$ \\
\hline
\end{tabular}

$R P$ routine parameters, $O P$ optimized parameters, $W M$ white matter, GM grey matter

"Wilcoxon signed rank test, $p<0.05$ for all centres; ${ }^{* *}$ Wilcoxon signed rank test, $p<0.05$ for 18 centres; ${ }^{* * *}$ Pitman test, $p<0.05$

have an important effect on signal-to-noise ratio of whole body imaging, but that effect is negligible on smaller objects such as brain structures [13].

Conversely, in images where both spatial resolution and $\mathrm{RC}$ were too low, we chose to use more iterations of the algorithm in order to enhance the spatial resolution of the images and to apply a Gaussian (FWHM between 2 and $4 \mathrm{~mm}$ ) post-reconstruction smoothing filter to the images. The pixel spacing was between 1 and $3 \mathrm{~mm}$ in all optimized images.

\section{Improving contrast recovery and dispersion of RC values}

With optimized parameters, the RC significantly improved for the cold spheres, but not for the hot spheres, of close diameter. That difference between cold and hot spheres is partly related to the presence of the sphere walls, which are intrinsically cold. These walls affect the quantification to a greater extent in hot spheres than in cold spheres. Such a cold wall is specific to the phantom. One should also note that the optimized $\mathrm{RC}$ was higher in the hot spheres than in the cold spheres of similar diameter. The 
quantification in cold objects is complex, depending not only on spatial resolution but also on scatter correction and spatial sampling [14, 15], and of the non-negativity constraint of the statistical reconstruction algorithm MLEM without a specific description [16].

We also significantly reduced the variability of $\mathrm{RC}$ in four of the six spheres of the Jaszczak phantom. As shown in Figs. 3 and 4, this reduction of variability was mostly due to the suppression of outliers by discarding PSF modelling reconstruction algorithms.

It should be emphasized that the RC measurements were obtained with in-house software, which was developed with great care to ensure reproducibility and precision. In particular, the VOIs were not drawn directly on the PET images but were automatically segmented on CT images and optimized with knowledge from the phantom specifications.

Improving quantification and variability in small brain structures and image uniformity We checked the quantification in the striatum of the Hoffman brain phantom and observed an increase in activity concentration measured in the putamen and caudate, when compared to non-optimized images. We also significantly reduced after the optimization the dispersion of values for putamen, caudate, and grey-matter-to-white-matter ratios.

Finally, the good uniformity of the images across the field of view was confirmed by the ratio of right-to-left activity concentration and anterior-to-posterior activity concentration measured on the Hoffman brain images. Additionally, as expected, the choice of the reconstruction mode parameters had no impact on the ratio of activity concentration in the right-to-left and frontal-to-occipital regions of interest. Those ratios should not be affected by the spatial resolution. However, they are likely affected by low frequency variation resulting from scatter, uniformity, and attenuation corrections. Attempts to take into account these effects before pooling data sets from multiple centres were suggested by Joshi et al. [1], but the results were not considered as convincing by the authors. In the present work, the compensation for scatter, random coincidences, and attenuation was performed using the manufacturer's latest techniques, with slight differences in the implementation for the different scanner models. The Hoffman phantom also enabled us to verify that the quantification in typical small structures of the brain, such as the caudate, was improved after the reconstruction optimization.

Image spatial resolution and signal-to-noise ratio

On average, image spatial resolution was slightly improved, despite a marked decrease for the centres equipped with reconstruction algorithms including TOF and PSF modelling. This improvement is likely due to the increase in the number of iterations in the other centres. As expected, this improvement is counterbalanced by a decrease of the signal-to-noise ratio. We checked with Hoffman phantom acquisitions that this reduction did not impact the visual aspect of the images.

\section{Variability across centres}

The variability across centres was reduced but not minimized by this work. For that, it would have been necessary to apply post-filtering tailored per site according to ISR. We 
deliberately chose not to do this in order to preserve the spatial resolution. This latter goal was achieved for the 18 centres without PSF modelling reconstruction.

In order to account for the residual variability across centres, we are currently designing a statistical model that takes into account the centre effect (as assessed by the phantom studies). Several other parameters have been shown to influence the variability of the measurements across centres, including injected dose per kilogram, delay between the injection and the scan acquisition, patient positioning, weight, and glycaemia, and these have been extensively discussed by Boellard [17, 18]. We will add to the statistical model these additional acquisition and patient-related parameters (as assessed by an ancillary monocentric study involving 300 subjects), and we will test it on the MEMENTO cohort.

If the statistical model happens not to be sufficiently effective, our approach could be updated in a second phase according to the strategy proposed by Lasnon et al. [19]. The latest advances in reconstruction schemes could be used to reconstruct images together with an adequate post-reconstruction filtering, offering the best compromise in image quantification for multicentre studies.

\section{Conclusions}

This work was undertaken by CATI's team in charge of harmonization, quality check, and analysis of multicentre brain PET or SPECT acquisitions. The proposed procedure for PET imaging optimization enabled the production of images with a more homogenous spatial resolution across the centres. The spatial resolution was also preserved thanks to the use of statistical image reconstructions with iterations $\times$ subsets of at least 50, a Gaussian smoothing post-reconstruction filter with a FWHM between 2 and $4 \mathrm{~mm}$, and a pixel spacing between 1 and $3 \mathrm{~mm}$. The uniformity across the fields of view of the scanners was good.

We provided recommendations to each centre for minimizing the influence of other factors such as injected dose and interval post-injection. In addition, centres were qualified after the analysis of the images of a first test patient. CATI has now received more than 3000 PET images.

This step of optimization of the image characteristics acquired at different centres will thus allow us to account for the residual variability, which could be handled in the final statistical analysis using adequate modelling.

\section{Ethical approval}

This article does not contain any studies with human participants or with animals performed by any of the authors.

Competing interests

MO Habert received hononaria from GE healthcare as a consultant and from PIRAMAL and ROCHE as a speaker. S Marie has no potential conflict of interest to declare. $\mathrm{H}$ Bertin has no potential conflict of interest to declare. JB Martini has no potential conflict of interest to declare. M Reynal has no potential conflict of interest to declare. M Diallo has no potential conflict of interest to declare. A Kas received honoraria from PIRAMAL and ROCHE as a speaker. R Trebossen has no potential conflict of interest to declare. 
acquisition. RT conceived of the study and participated in the study design. All authors read and approved the final manuscript.

\section{Acknowledgements}

We sincerely thank the staff from all departments of Nuclear Medicine who participated in the MEMENTO cohort study for their availability and their patience in spite of our numerous requests.

We also are grateful to Alain Giron and Marion Houot for their help with statistical analyses, and to the staff from Esprimed (www.esprimed.net), who were in charge of phantom acquisitions, for their efficiency and professionalism. Finally, we greatly appreciate the support of Philips, GE Healthcare, and Siemens engineers in determining and setting up the optimized reconstruction parameters.

\section{Funding}

The French Foundation Plan Alzheimer supported this work.

\section{The CATI Nuclear Medicine network includes as follows:}

Pascal Bailly (CHU Sud, Amiens), Sébastien Balduyck (Hôpital Purpan, Toulouse), Francis Bouchet (CHU Angers), Hatem Boulahdour (Hôpital Jean Minjoz, Besançon), David Bourhis (CHRU Morvan, Brest), Thomas Carlier (Hôpital Nord Laennec, Nantes), Jacques Darcourt (Centre Antoine Lacassagne, Nice), Marielle Decousus (Hôpital Nord, Saint-Etienne), Delphine De Verbizier Lonjon (Hôpital Gui de Chauliac, Montpellier), Inna Dygai-Cochet (Centre George François Leclerc, Dijon), Élise Enderlin (Hôpital de Hautepierre, Strasbourg), Karim Farid (Centre Cardiologique du Nord, SaintDenis), Bardia Farman (Hôpital La Timone, Marseille), Philippe Fernandez (Hôpital Xavier Arnozan, Pessac), Pacôme Fosse (CHU Angers), Marjolaine Fourcade (Hôpital Gui de Chauliac, Montpellier), Christelle Gallais (CHU Poitiers - TEP GIE), Céline Gallazzini-Crepin (Hôpital Albert Michallon, Grenoble), Éric Guedj (Hôpital La Timone, Marseille), Sébastien Hapdey (Centre Henri Becquerel, Rouen), Anne Hitzel (Hôpital Purpan, Toulouse), Claude Hossein-Foucher (Hôpital Huriez, Lille), Laetitia Imbert (CHU Nancy-Brabois), Anthony Kelly (Centre Jean Perrin, Clermont-Ferrand), Pierre Malick Koulibaly (Centre Antoine Lacassagne, Nice), Fabien Maurel (CHU Nice), Marc-Etienne Meyer (CHU Sud, Amiens), Corinne Millardet (Centre Jean Perrin, Clermont-Ferrand), Izzie Jacques Namer (Hôpital de Hautepierre, Strasbourg), Amandine Pallardy (Hôpital Nord Laennec, Nantes), Catherine Pérault (Hôpital Bretonneau, Tours), Rémy Perdrisot (CHU Poitiers - TEP GIE), Yolande Petegnief (Hôpital Jean Minjoz, Besançon), Gabriel Pop (Hôpital Avicenne, Bobigny), Nathalie Prevot-Bitot (Hôpital Nord, Saint-Etienne), Mathieu Queneau (Centre Cardiologique du Nord, Saint-Denis), Solenne Querellou (CHRU Morvan, Brest), Micheline Razzouk-Cadet (CHU Nice), Ghislaine Reboulet (Hôpital Albert Michallon, Grenoble), Maria-Joao Santiago-Ribeiro (Hôpital Bretonneau, Tours), Pierre Vera (Centre Henri Becquerel, Rouen), Antoine Verger (CHU Nancy-Brabois), Maximilien Vermandel (Hôpital Huriez, Lille), Jean-Marc Vrigneaud (Centre George François Leclerc, Dijon), Marie-Joséphine Waryn (Hôpital Avicenne, Bobigny), and Michel Zanca (Hôpital Gui de Chauliac, Montpellier).

\section{Author details}

'Sorbonne Universités, UPMC Univ Paris 06, CNRS, INSERM, Laboratoire d'Imagerie Biomédicale, F-75013 Paris, France. ${ }^{2}$ Centre pour I'Acquisition et le Traitement des Images (www.cati-neuroimaging.com), Paris, France. ${ }^{3}$ AP-HP, Hôpital Pitié-Salpêtrière, Département de Médecine Nucléaire, F-75013 Paris, France. ${ }^{4}$ CEA-Service Hospitalier Frédéric Joliot, F-91401 Orsay, France. ${ }^{5}$ Faculté de Médecine - Laboratoire d'Imagerie Biomédicale, 91 Boulevard de l'Hôpital, F-75013 Paris, France.

Received: 11 November 2015 Accepted: 9 February 2016 Published online: 05 April 2016

\section{References}

1. Joshi A, Koeppe RA, Fessler JA. Reducing between scanner differences in multi-center PET studies. Neurolmage. 2009;46(1):154-9.

2. Herholz K, Salmon E, Perani D, Baron C, Holthoff V, Frölich L, Schönknecht P, Ito K, Mielke R, and Kalbe E. Discrimination between Alzheimer dementia and controls by automated analysis of multicenter FDG PET. Neurolmage. 2002;17(1):302-16.

3. Mosconi L, Tsui WH, Herholz K, Pupi A, Drzezga A, Lucignani G, Reiman EM, Holthoff V, Kalbe E, Sorbi S, DiehlSchmid J, Perneczky R, Clerici F, Caselli R, Beuthien-Baumann B, Kurz A, Minoshima S, and de Leon MJ. Multicenter standardized 18 F-FDG PET diagnosis of mild cognitive impairment, Alzheimer's disease, and other dementias. J Nucl Med. 2008;49(3):390-8.

4. Geworski L, Knoop BO, de Wit M, Ivancević V, Bares R, Munz DL. Multicenter comparison of calibration and cross calibration of PET scanners. J Nucl Med. 2002;43(5):635-9.

5. Westerterp M, Pruim J, Oyen W, Hoekstra O, Paans A, Visser E, van Lanschot J, Sloof G, and Boellaard R. Quantification of FDG PET studies using standardised uptake values in multi-centre trials: effects of image reconstruction, resolution and ROI definition parameters. Eur J Nucl Med Mol Imaging. 2007;34(3):392-404.

6. Scheuermann JS, Saffer JR, Karp JS, Levering AM, Siegel BA. Qualification of PET scanners for use in multicenter cancer clinical trials: the American College of Radiology Imaging Network experience. J Nucl Med. 2009;50(7): 1187-93.

7. Varrone A, Asenbaum S, Vander Borght T, Booij J, Nobili F, Någren K, Darcourt J, Kapucu OL, Tatsch K, Bartenstein P, Van Laere K, and European Association of Nuclear Medicine Neuroimaging Committee. EANM procedure guidelines for PET brain imaging using [18 F]FDG, version 2. Eur J Nucl Med Mol Imaging. 2009;36(12):2103-10.

8. Ballard DH. Generalizing the Hough transform to detect arbitrary shapes. Pattern Recogn. 1981;13(2):111-22.

9. Prieto E, Martí-Climent JM, Arbizu J, Garrastachu P, Domínguez I, Quincoces G, García-Velloso MJ, Lecumberri P, Gómez-Fernández M, Richter JA. Evaluation of spatial resolution of a PET scanner through the simulation and experimental measurement of the recovery coefficient. Comput Biol Med. 2010;40(1):75-80. 
10. Pitman EJG. A note on normal correlation. Biometrika. 2013;31:9-12.

11. Weiner MW, Veitch DP, Aisen PS, Beckett LA, Cairns NJ, Green RC, Harvey D, Jack CR, Jagust W, Liu E, Morris JC, Petersen RC, Saykin AJ, Schmidt ME, Shaw L, Shen L, Siuciak JA, Soares H, Toga AW, Trojanowski JQ, and Alzheimer's Disease Neuroimaging Initiative. The Alzheimer's Disease Neuroimaging Initiative: a review of papers published since its inception. Alzheimers Dement. 2013;9(5):e111-94.

12. Zeng GL. Gibbs artifact reduction by nonnegativity constraint. J Nucl Med Technol. 2011;39(3):213-9.

13. Conti M. Focus on time-of-flight PET: the benefits of improved time resolution. Eur J Nucl Med Mol Imaging. 2011; 38:1147-57.

14. Stute S, Comtat C. Practical considerations for image-based PSF and blobs reconstruction in PET. Phys Med Biol. 2013:58(11):3849-70.

15. Bendriem B, Dewey SL, Schlyer DJ, Wolf AP, Volkow ND. Quantitation of the human basal ganglia with positron emission tomography: a phantom study of the effect of contrast and axial positioning. IEEE Trans Med Imaging. 1991:10(2):216-22.

16. Van Slambrouck K, Stute S, Comtat C, Sibomana M, van Velden F, Boellaard R, and Nuyts J. Bias reduction for lowstatistics PET: maximum likelihood reconstruction with a modified Poisson distribution. IEEE Trans Med Imaging. 2015;34(1):126-36.

17. Boellaard R, O'Doherty MJ, Weber WA, Mottaghy FM, Lonsdale MN, Stroobants SG, Oyen WJ, Kotzerke J, Hoekstra OS, Pruim J, Marsden PK, Tatsch K, Hoekstra CJ, Visser EP, Arends B, Verzijlbergen FJ, Zijlstra JM, Comans EF, Lammertsma AA, Paans AM, Willemsen AT, Beyer T, Bockisch A, Schaefer-Prokop C, Delbeke D, Baum RP, Chiti A, and Krause BJ. FDG PET and PET/CT: EANM procedure guidelines for tumour PET imaging: version 1.0. Eur I Nucl Med Mol Imaging. 2010; 37(1):181-200.

18. Boellaard R. Need for standardization of 18 F-FDG PET/CT for treatment response assessments. J Nucl Med. 2011; 52 Suppl 2:93S-100S

19. Lasnon C, Desmonts C, Quak E, Gervais R, Do P, Dubos-Arvis C, and Aide N. Harmonizing SUVs in multicentre trials when using different generation PET systems: prospective validation in non-small cell lung cancer patients. Eur J Nucl Med Mol Imaging. 2013;40(7):985-96.

Submit your manuscript to a SpringerOpen ${ }^{\circ}$ journal and benefit from:

- Convenient online submission

Rigorous peer review

- Immediate publication on acceptance

- Open access: articles freely available online

High visibility within the field

- Retaining the copyright to your article 